Article

\title{
Study on the Hazard Limitation of Hybrid Circuit Breaker Actuator Operation
}

\author{
Piotr Jankowski * and Janusz Mindykowski \\ Department of Marine Electrical Power Engineering, Gdynia Maritime University Poland, \\ 81-225 Gdynia, Poland; j.mindykowski@we.am.gdynia.pl \\ * Correspondence: p.jankowski@we.am.gdynia.pl; Tel.: +48-58-5586440
}

Received: 21 December 2017; Accepted: 8 February 2018; Published: 11 February 2018

\begin{abstract}
The paper deals with a key issue, how to limit the hazard and assure reliability, safety and repeatability of operation of selected critical devices protecting electrical power systems. The main research is focused on the incorrect operation of hybrid switches caused by random disturbances of the thyristor gate signals in the electrodynamic drive coil power supply circuit. Firstly, a pilot experiment on the operation of hybrid circuit breaker actuators under random disturbance conditions was carried out and the results are presented. Next, an in-depth operation analysis, based on experimental and simulation research, according to setting conditions of the circuit breaker actuator parameters such as capacitance, voltage and switching time was performed. A simulation study with the use of a field-circumferential model in the Ansys Maxwell program of electrodynamic propulsion of rectilinear motion, which enables energy transfer from the capacitor bank to the actuator coil power supply at selected time intervals, was executed. The study analysed the impact of the discontinuity of energy transfer on the dynamic properties of the actuator and some recommendations for designers and users on how to limit this hazard are formulated.
\end{abstract}

Keywords: hybrid circuit breaker; electrodynamic actuator; hazard limitation; discontinuous energy transfer; ultra-rapid displacement; model in Maxwell environment; experimental research

\section{Introduction}

The article concerns the analysis of processes and the operation of hybrid switches [1,2] related to the arcless switching off of short-circuit currents in power circuits operating in difficult environmental conditions [3,4]. In the conditions under consideration, the main cause of disruption of the power system operation is a dynamic short circuit, but one cannot omit such factors as vibrations, lightning, or changing weather conditions. Such conditions occur, among others, in ship electric power systems or electric traction railway systems [4,5]. Due to the fact that the operation of switching off short-circuit currents refers to emergency states and is essential for the operational safety of the considered power system, the devices performing these operations, usually hybrid switches, belong to the group which can be referred to as critical devices for electric power system operation.

Because the critical devices fulfill a vital role in the operation of the considered systems, they should ensure the reliability, safety and repeatability of the realized operations. A principal module of the hybrid circuit breaker is an electrodynamic propulsion-actuator, responsible for the safe and correct switching off of the controlled circuit.

In the solution presented in this article, an electrodynamic propulsion-actuator is realized as the inductive dynamic drive (IDD). The IDD (Figures 1 and 3) is an integral part of a current limiting interrupting device (CLID) (Figure 1a,b), in which this actuator works synchronously with semiconductor elements [5,6].

The discharge of the capacitor battery (of the IDD) occurs in a circuit of low resistance and inductance so that through the coil flows a large current generating a large magnetic field. A variable 
magnetic field induces (in the disc surface layer) eddy currents, which generate impulsive force (displacing disc) when interacting with the current coil. At this time, the energy-absorbing element (e.g., varistor) minimizes the short-circuit current to the value at which the drive, spacing the contacts apart, allows for the arcless switching off of the short circuit.

It follows that the electrodynamic actuator must be characterized by repeatability and a suitable duration of action [7-9]. The necessity of complying with these requirements is a reason for the continuous research and study of the impact of influencing disturbing factors for electrodynamic actuator-propulsion operation. The undertaken research and study results from the aforementioned industrial application experiences include harsh environment applications such as sea or railway conditions on the one hand, and, on the other hand, laboratory investigations aimed at the further development of existing solutions. In some cases a malfunction of electrodynamic actuator operation consisting in a discontinuity of energy transfer from discharged capacitors to the drive coil has been noted. This phenomenon has been occurring randomly, so it can be considered a kind of hazard of electrodynamic actuator operation.

Basically, the trigger circuit (Silicon Controlled Rectifier -SCR thyristor or Insulated Gate Bipolar Transistor-IGBT) in the drive coil power supply circuit should generate an uninterrupted signal (Figure 4a), however in real industrial systems (e.g., industrial systems in sea conditions) the occurrence of such disturbances is inevitable, especially in the case of the appearance of a large short-circuit pulse (Figure 4b). Additionally, the results of the pilot experimental research concerning ultra-rapid drives also confirm the stochastic appearance of disturbing signals, that is, discontinuities in the energy transfer to the drive coil, understood as coil current flow interruption.

The aforementioned circumstances were a reason to order (by the CLID module users) the authors' research team to carry out experimental research which would unambiguously explain the cause of the previously mentioned energy transfer discontinuities and the consequences of their affecting on the correctness of operation of the IDD actuator in the CLID module (Figure 1).

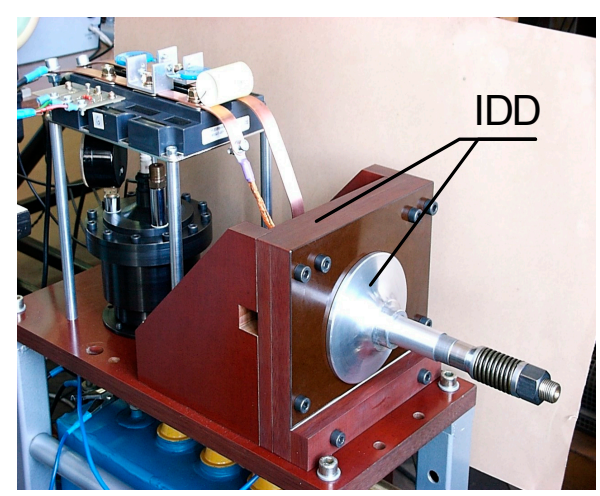

(a)

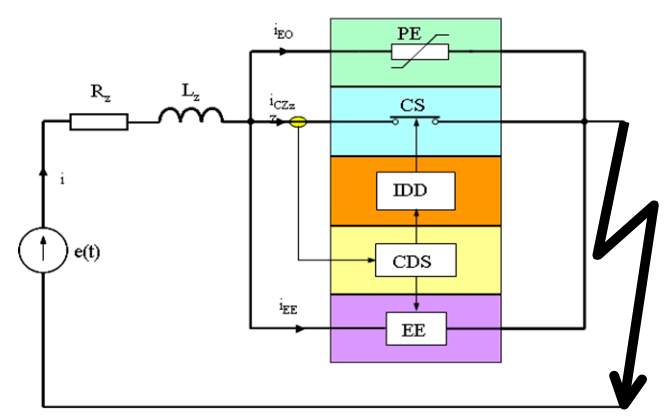

(b)

Figure 1. Current limiting interrupting device (CLID): (a) CLID photo (b) Block diagram.

In this context, a basic problem to solve is to identify the reasons and determine the conditions of occurrence of the hazardous operation of an electrodynamic propulsion-actuator, as well as to investigate the consequences of these phenomena and mitigation measures. A novelty of this paper concerns the carrying out of the experiment and simulation research, resulting in reasons explaining the incorrect operation of the hybrid switch drive, and elaboration of the authors' new field-circumferential model of rectilinear propulsion, enabling the analysis of the electrodynamic actuator operation under discontinuous energy transfer to the drive coil. This analysis is related to and focused on the examination of a working hypothesis: in the real ultra-rapid drives, a random, stochastic disturbing signal can appear in a trigger circuit and cause discontinuities in the energy transfer to the drive coil. For a better understanding of the contents of this paper, a flow-chart of 
the investigation carried out is shown in Figure 2, in order to effectively demonstrate the proposed experimental and simulation research.

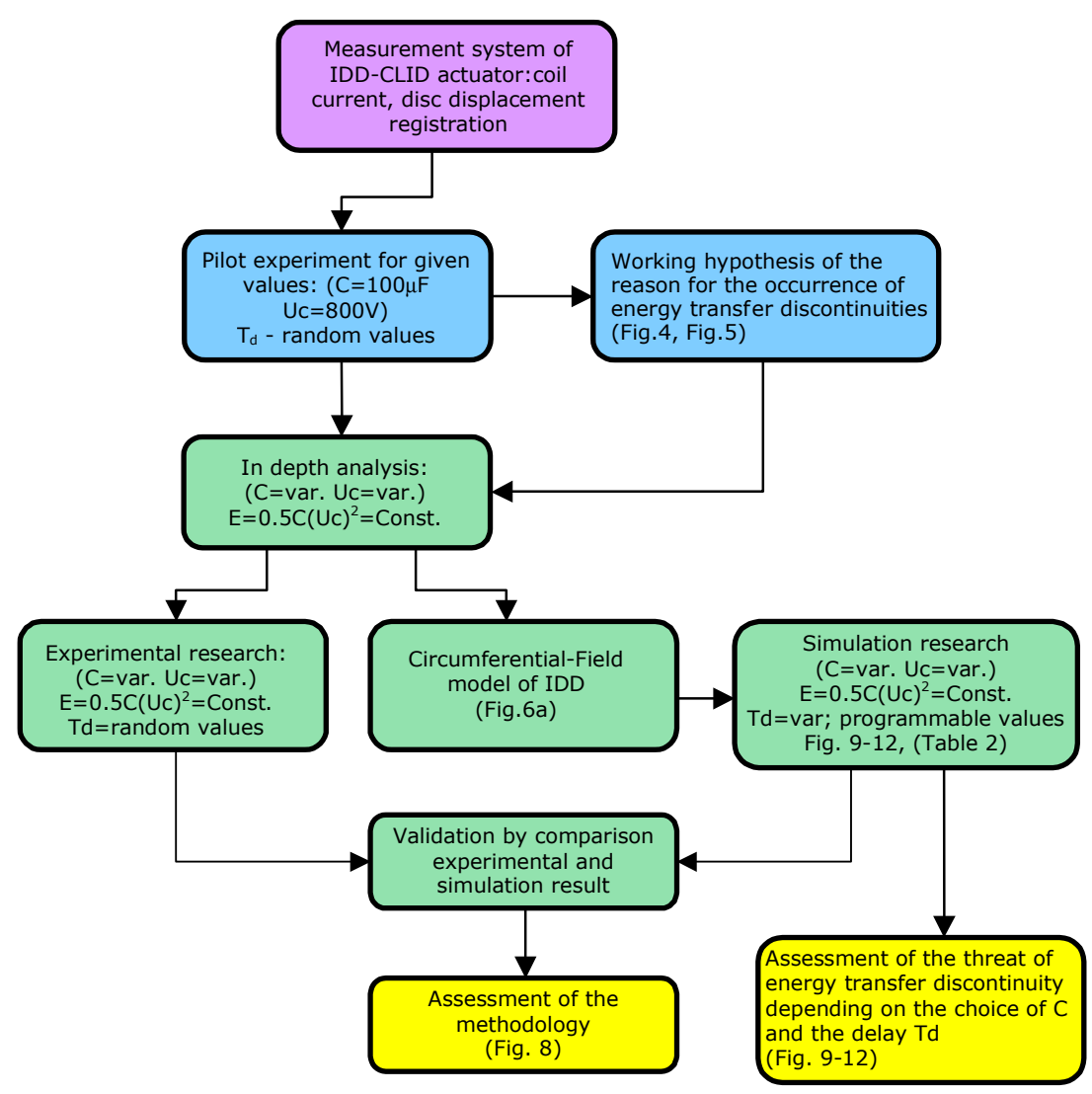

Figure 2. Flow-chart of the performed investigation.

To explain the reasons and nature of the appearing discontinuities, the standard example of the CLID device equipped with inductive dynamic drive (IDD) and supplied from the capacitor with a capacity of $C=100 \mu \mathrm{F}$ charged to the initial voltage value, which equals $U c=800 \mathrm{~V}$, was used. The applied measurement system (Figure 3a) enables the registration of the drive coil current and disc displacement. For the measurement system of the IDD (Figure 3), experimental pilot research was carried out. This research confirmed the appearance of the discontinuous flow of the coil current. Simultaneously, a gate signal in the drive coil power supply circuit was registered. It was observed that the appearance of coil current discontinuity (Figure 5a) was accompanied by randomly appearing discontinuity of the gate signal current (Figure $4 \mathrm{~b}$ ). Therefore, the previously-cited working hypothesis was formulated. In a further step, referred to by the authors an in-depth analysis, extended experimental research was continued to look for other values of capacitances $C$ and voltages $U c$ that comply all the time with the condition of the constant value of the initial energy $E$ of the capacitor battery. Within the framework of an in-depth analysis, in parallel to physical experiments, a field-circumferential model of the IDD drive was built and applied. Due to this model, a simulation of the moment of an interruption appearance in the gate current circuit expressed by delay time $\mathrm{Td}$, as well as an examination of the influence of this moment on the current continuity in the drive coil, and more, on the dynamic properties of the IDD drive expressed by disc displacement, was possible.

Following this chapter, the rest of this article is structured in this way: Section 2 describes the experimental study of the IDD dedicated measurement system, Section 3 presents the field circumferential model of rectilinear propulsion, and Section 4, fundamental for this paper, is devoted to 
the analysis of an influence of discontinuous energy transfer on the electrodynamic propulsion-actuator dynamic properties. The last section is a conclusion of the study.

\section{Experimental Study}

The description of the measuring system (Figure 3a), allowing for the registration of the coil current and the displacement of the drive (IDD) using the optical sensor (optimeter) was presented in detail in $[7,8]$. The power supply circuit of the coil is closed by controlled valve (transistor or thyristor with diode) so that one can conclude that the release of this valve occurs almost immediately after the detection of a short circuit. The gate circuit in the tested drive model can generate a continuous signal flowing in a short time of the whole process (less than a few milliseconds). Hence, it can be easily implemented as a (Resistive-Capacitive) RC circuit for discharging a small capacitor (Figure 3b). On the other hand, in a laboratory environment, a contactor is often used to initiate the discharge of this gate circuit. As a result of many tests (pilot experimental research) carried out in the measurement system (Figure 3), it turned out that the correct gate signal was obtained with a similarly random frequency (with similar probability distribution), as the disrupted signal (Figure 4). The obvious explanation for this is the appearance of the contact bounce of the contactor. Such a disturbed gate circuit signal is also the cause of discontinuous energy transfer from the capacitor bank to the coil (red waveform Figure 5a). At the same time, it should be emphasized that in industrial conditions, especially marine (e.g., stormy) conditions, such a disturbance of the gate control signal of low power at the moment of short-circuiting impulse may also appear during the operation of the CLID system, with a contactor-less solution.

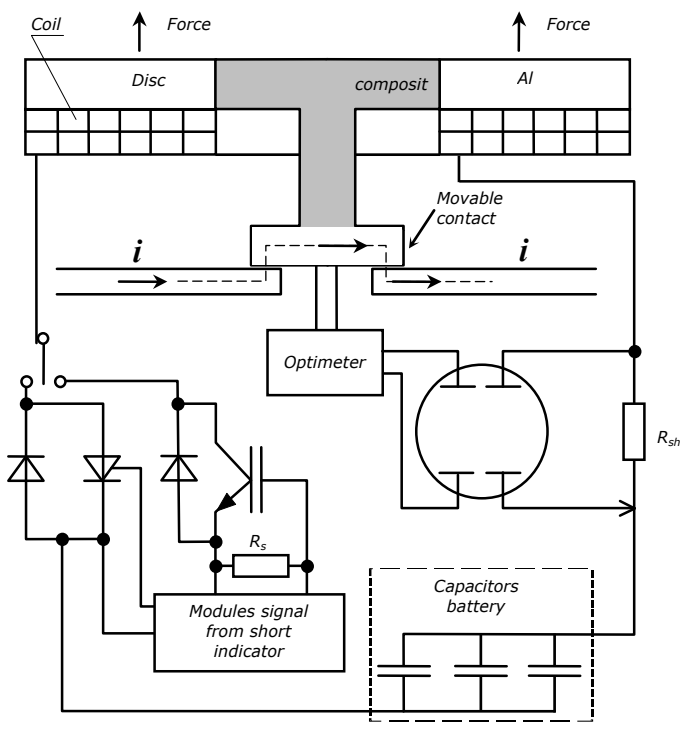

(a)

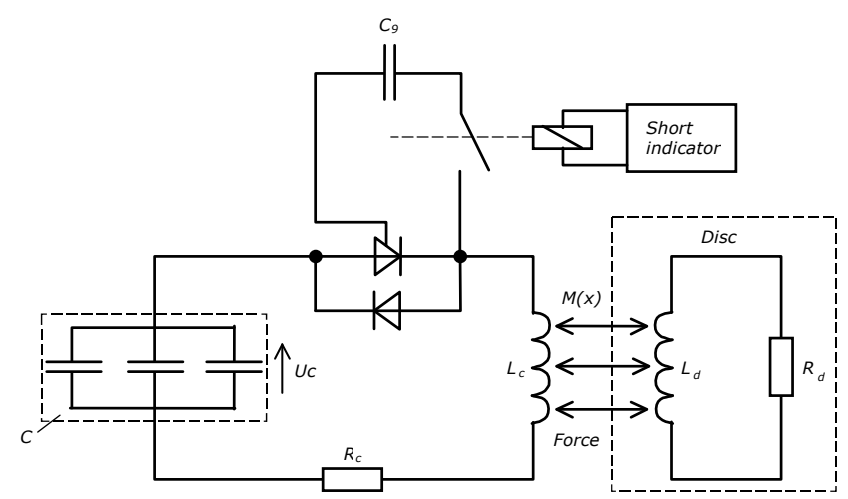

(b)

Figure 3. System of IDD (a) Measurement scheme (b) Electric scheme-simple realization of the gate circuit.

Due to the modular design of the capacitor bank (Figure 3a) it is possible to change the total capacitance $(C)$ of the capacitors, while maintaining the initial energy $E$ of the battery by changing the initial voltage $U c$ accordingly:

$$
E=\frac{C\left(U_{c}\right)^{2}}{2}=\text { Const. }
$$

where $C$ is the total capacitance of the capacitor battery, and $U_{c}$ is the initial voltage value.

Presuming that electrodynamic drive efficiency depends on the capacitance in a small measure, then on the basis of the energy conservation law (the electric field energy corresponds to the product 
of efficiency and disc kinetic energy), it might be supposed that the value of the total battery capacity used does not have a significant impact on the dynamic characteristics of the drive (maximal value of displacement in the given set time), if the condition of the initial energy of the capacitor bank is retained. However, the further experimental research carried out for a 10 times higher capacity of the $C$ capacitors and reduced (respectively for the condition $E=$ Const.) voltage value showed that the drive disc reached a greater displacement range than previously (Figure $5 b$ ). This time, the energy transfer maintained continuity, despite the occurrence of disturbances in the gate circuit in the initial period (Figure $4 \mathrm{~b}$ ), which means that the disruption disappeared before the thyristor switched back on (blue waveform Figure 5a). In order to clarify whether the increased value of the displacement range in case of the battery capacitance increase (at energy $E=$ Const.) is caused by the continuity of energy transfer to the drive coil, further tests were carried out, both simulative using the new IDD model as well as experimental.

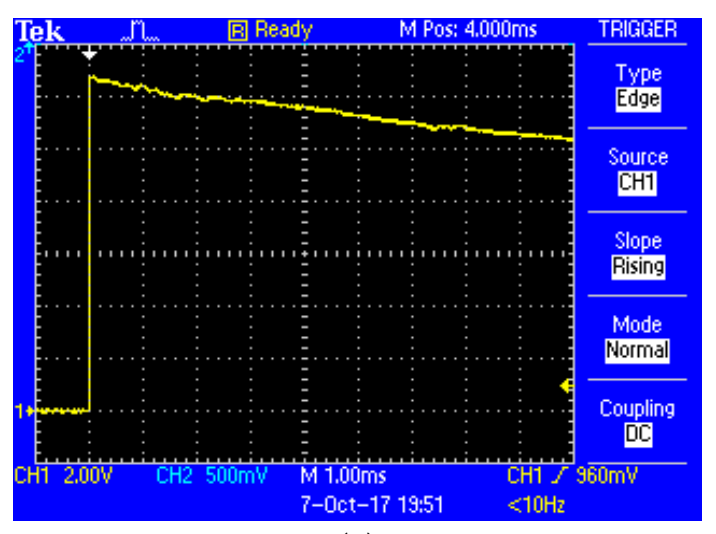

(a)

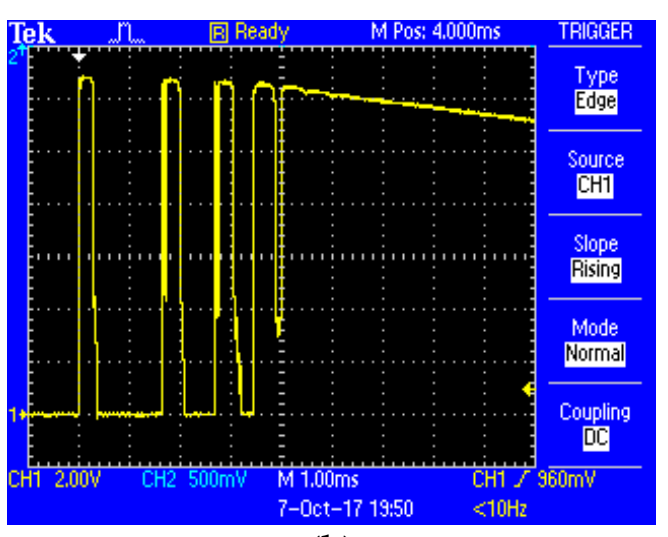

(b)

Figure 4. Oscillograms of the thyristor gate: (a) Continuous gate signal (b) Disturbed gate signal.

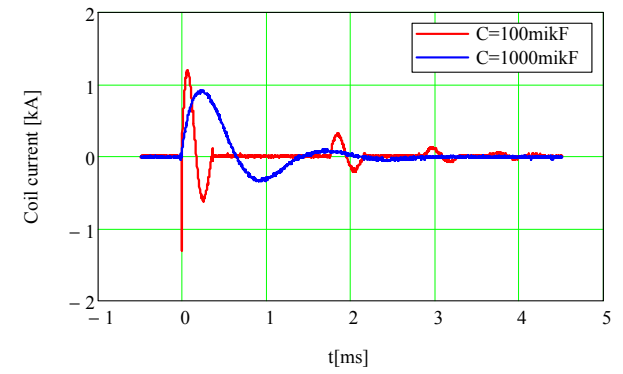

(a)

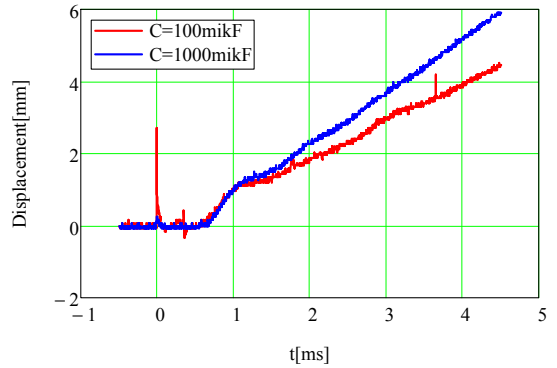

(b)

Figure 5. Registration of waveforms (at disturbed gate signal from Figure $4 \mathrm{~b}$ ) for $C=100 \mu \mathrm{F}$ red line, $C=1000 \mu \mathrm{F}$ blue line: (a) Coil currents (b) Displacements.

\section{Field-Circumferential Model of Rectilinear Propulsion}

In order to examine the above effect of the discontinuous transfer of energy from the capacitor on the dynamic properties of propulsion, it was decided to carry out simulation tests in the field model in which controlled switches in the power supply of the coil circuit will be able to close the circuit at a selected time. A 2D (two-dimensional) propulsion model was realized in the Maxwell program, which takes into account the movement of the disc under the influence of the electrodynamic interaction force. The elementary electrodynamic interaction force between the coil and the disc is proportional 
to the cross product of the current density and flux density generated by the coil current. Hence, the instantaneous force function $F(t)$ is obtained on the basis of the following volume integral [10-14].

$$
F(t)=\iiint_{V_{d i s c}} \mathbf{J}(t) \times \mathbf{B}(t) d V_{d i s c}
$$

Then the function of the disc displacement $x(t)$ can be determined using the following formula:

$$
x(t)=\frac{1}{m} \int_{0}^{t}\left(\int_{0}^{t}\left(\iiint_{V_{\text {disc }}} \mathbf{J}(t) \times \mathbf{B}(t) d V_{\text {disc }}-F_{\text {load }}\right) d t\right) d t
$$

Such an IDD drive model was implemented and described in detail in $[9,15]$. Due to the fact that the implementation of the circumferential part of the model existing so far makes it impossible to simulate discontinuous energy transfer, appropriate modifications have been made.

The main goals of IDD model modification are:

- the field-circuit model of drive executed in the Maxwell program along with an accompanying external circuit containing controlled switches.

- the simulation and analysis results in the coil current, force and the displacement drive for different moments of activation switches.

A model modified this way enables the simulation of a discontinuous coil current flow and discharging voltage of the capacitor battery, resulting in energy flow interruptions between capacitor and coil, in other words, discontinuity of the energy transfer.

The realization of the simulation research presented in this article is shown in diagram form in Figure 6a.

By appropriately setting the control signal switches S_ON1 and S_ON2, simulation results of coil current, forces, and hence the disc displacements for the continuous and discontinuous energy transfer from the capacitor were obtained. The model constitutes half of the cross-section of the coil-disc system, therefore cylindrical symmetry of the drive was used (Figure $6 b$ ). The number of meshing elements was limited to 20,000, into which the whole area was discretized, which was the guarantee of the convergence of the obtained results. As a boundary condition, a potential vector on the boundary area (region) with the value of zero was assumed.

Figure $6 \mathrm{~b}$ shows a screen from the propulsion model executed in the Maxwell program representing half of the drive in the cylindrical system with meshing into elements. Magnetic field distribution (for the initial moment) shown in this figure presents a strong phenomenon of skin effect due to the rapidly increasing current. Since the coil in the model was associated with winding, then (in the Ansys-Maxwell program $[16,17])$ it is possible to attach to the external circuit. Parameters defining the dimensions of the coil and the disc of the drive as well as capacity of the capacitor battery are included in Table 1. Because the model uses an object defined by the program as a coil, which must be linked to winding, inductance is calculated by the model in every numerical step. 


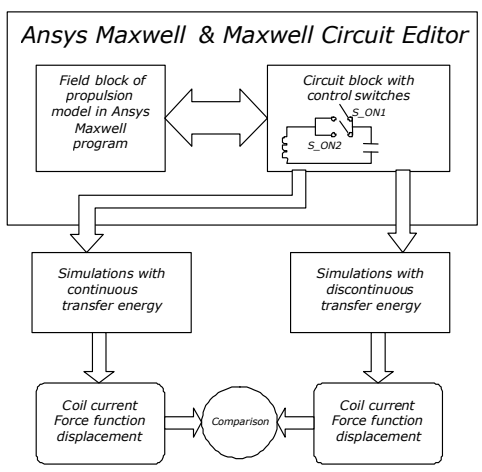

(a)

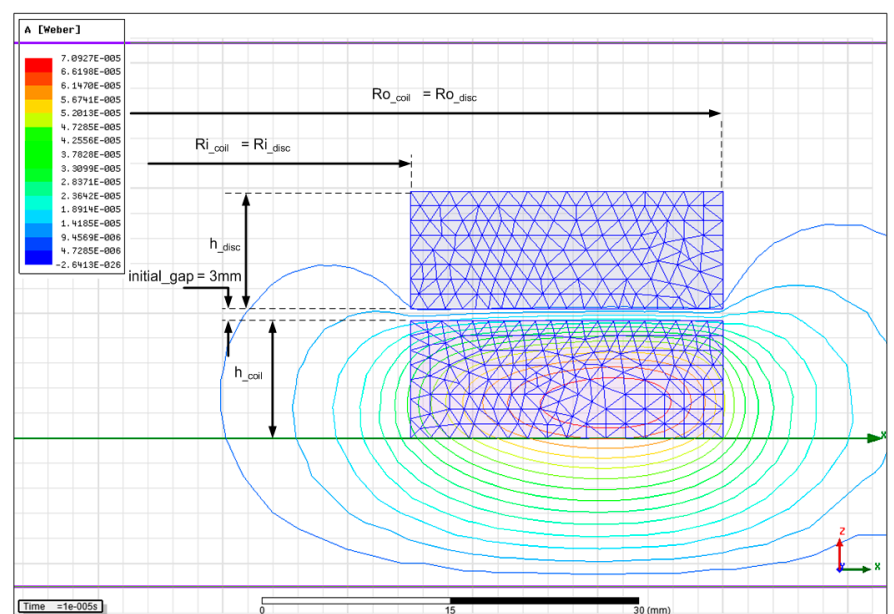

(b)

Figure 6. IDD model in the Ansys-Maxwell program: (a) Block scheme of the presented simulation examinations, (b) Flux density results in actuator model in Maxwell.

Table 1. Propulsion (IDD) parameters.

\begin{tabular}{ccc}
\hline Parameter & Symbol & Magnitude \\
\hline Inner coil radius & $R i_{\text {ccoil }}$ & $45 \mathrm{~mm}$ \\
Outer coil radius & $R o_{-c o i l}$ & $70 \mathrm{~mm}$ \\
Coil thickness & $h_{-c o i l}$ & $9.375 \mathrm{~mm}$ \\
Wires number & $N$ & 24 \\
Inner disc radius & $R i_{-}$disc & 45 \\
Outer disc radius & $R o \_d i s c$ & $70 \mathrm{~mm}$ \\
Disc thickness & $h_{-d i s c}$ & $9.375 \mathrm{~mm}$ \\
Capacitor capacitance & $C$ & $100 \mu \mathrm{F} / 1000 \mu \mathrm{F}$ \\
\hline
\end{tabular}

In contrast to inductance, the resistance of the coil is not calculated by the Maxwell program, so it was required to be estimated, which was done on the basis of the formula where the coefficient $k$ includes the impact of the skin effect:

$$
R_{\text {coil }}=\frac{4 k_{\text {skin_eff }}}{\gamma_{c u} \cdot \pi \cdot\left(d_{\text {wire }}\right)^{2}} \sum_{c i=1}^{N}\left(2 \pi \cdot r_{c}(c i)\right)
$$

where $r_{c}(c i)$ is the radius of $c i$-th wire.

Skin effect ratio:

$$
k_{\text {skin_eff }}=\frac{|\Delta| d_{\text {wire }}}{4} \frac{b_{0}}{b_{1}} \cos \left(\beta_{0}-\beta_{1}+\frac{\pi}{4}\right)
$$

where

$d_{\text {wire }}$ is the wire diameter,

$b_{0}, \beta_{0}$ are the absolute value and argument of the modified first kind Bessel function, $b_{1}, \beta_{1}$ are the absolute value and argument of the modified second kind Bessel function, and

$$
|\Delta|=\sqrt{\omega \mu \gamma_{c u}}
$$

where $\omega$ is the current angular frequency, $\mu$ is the permeability, and $\gamma_{c u}$ is the conductivity.

The external circuit was built in the Ansys_Maxwell_Circuit_Editor subprogram (Figure 7a). After opening this sub-program the coil symbol appears as the first object, which represents the coil of 
the main program Maxwell. By including further elements we can create an outer circumference of the coil, which is then exported to the main model in Maxwell program. This circuit employs two parallel switches that are controlled by a periodic signal (Figure $7 \mathrm{~b}$ ). The parameters of the signal, i.e., the rise time $T_{r}$ and fall time $T_{f}$ and the resistance value $R_{\text {off }}$ and $R_{o n}$ of the switch in the off and on state in the block model (Figure 7a), one can set in such a way that operation of these connectors will be similar to the actual valve, such as a thyristor or a transistor. In the present study, very short times $\left(1 \times 10^{-15} \mathrm{~s}\right)$ were adopted for $T_{r}$ and $T_{f}$, that is, it is assumed that both valves have similar, almost perfect dynamic properties. The on-time $T_{d}$ of the first connector is set to zero, while the second connector on-time was adjusted depending on with what delay we want the capacitor to return the next portion of energy.

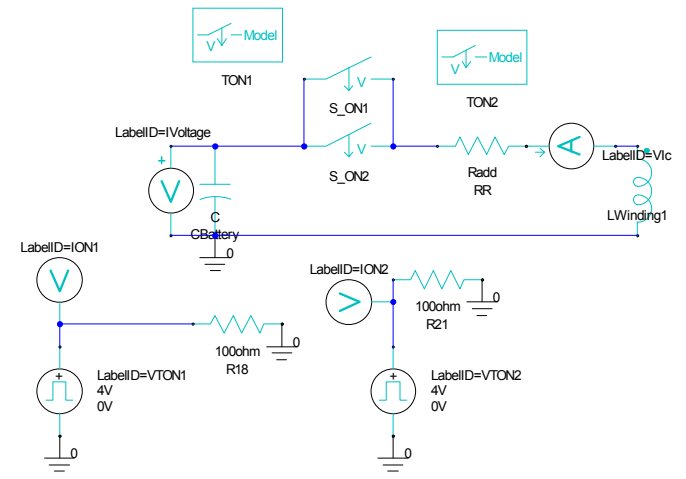

(a)

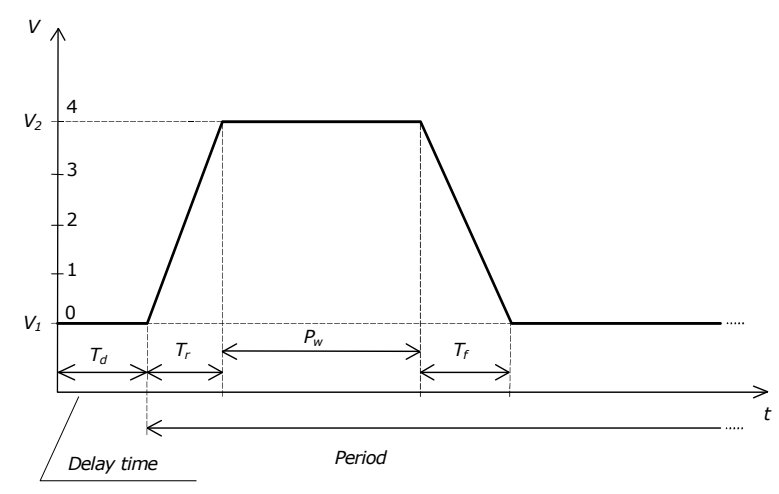

(b)

Figure 7. Modelling of the power supply of the drive coil (a) Outer circuit supplying of the coil (b) Shape of the trigger signal.

Preliminary simulation research for the validation of the IDD model has been compared with the experimental research for the propulsion parameters shown in Table 1 (Figure 8). This validation is based on the use of the field-circumferential model of rectilinear propulsion executed in the Maxwell program and the experiment made in the IDD measuring system presented in Section 2 (Figure 3a).

Figure 8a shows a comparison of the coil current waveform obtained in the field model with the course derived from the experiment with the bidirectional switch. Figure $8 \mathrm{~b}$ shows a similar comparison of the disc displacements received from the circumferential-field model and the experiment. The results illustrated in Figure $8 \mathrm{a}, \mathrm{b}$ are composed of two records, expressed by a solid line (experimental results) and a dash line (simulation results). The experiments and simulation research were carried out for the same capacity value $C=100 \mu \mathrm{F}$ and for the remaining IDD parameters listed in Table 1.

Based on the observation of Figure $8 \mathrm{a}, \mathrm{b}$ it is to be said that the comparison of coil currents proves very good convergence and hence the correctness of the constructed field-circumferential model. In the case of displacements, one can see the difference in the waveforms in the initial phase of the movement $(x<1 \mathrm{~mm})$, which can be explained by the fact that the presented drive model assumes, firstly, the ideal stiffness of its moving element (disc) and, secondly, the lack of any vibrations of measurement system, which is unavoidable [18]. 


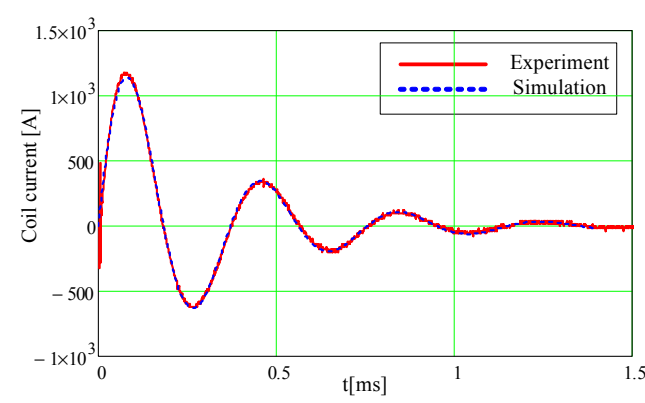

(a)

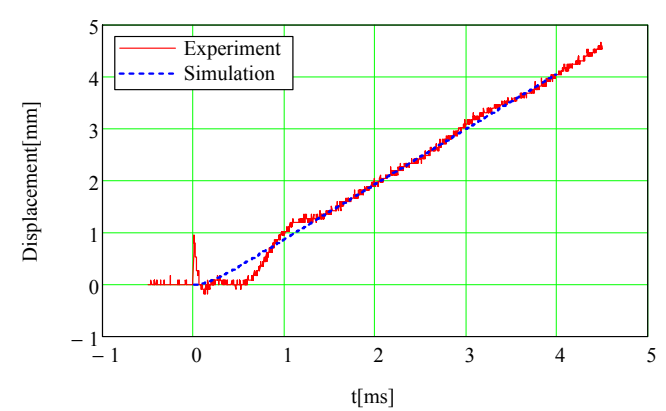

(b)

Figure 8. Comparison of results obtained from the experiment and simulation research for parameters from Table 1 at $C=100 \mu \mathrm{F}$ (a) coil current (b) disc displacement.

\section{Influence of Discontinuous Energy Transfer on Dynamic Properties of Propulsion}

Further studies were performed using the built field-circumferential model according to the scheme illustrated in Section 3 (Figure 6a) and cover cases presented in Table 2. Of the tests carried out for the present paper, three cases are shown in which the waveforms of the coil current, force and disc displacement for the continuous and discontinuous energy transfer from the capacitor were compared. The on-time $\left(\mathrm{Td}_{1}\right)$ of the first switch S_ON1 was set as zero (start simulation). The discontinuity was adjusted by the off-time $\left(\mathrm{Pw}_{1}\right)$ of the first switch S_0N1 and the on-time $\left(\mathrm{Td}_{2}\right)$ of the second switch S_0N2 (Figure 7b). The exact values of the on and off times for the presented study (three cases) are shown in Table 2.

Table 2. Switch parameters for the examined cases.

\begin{tabular}{|c|c|c|c|c|c|c|c|}
\hline & & $u_{c}$ & $C$ & $\mathrm{~T}_{\mathrm{d} 1}$ & $P_{w 1}$ & $\mathrm{~T}_{\mathrm{d} 2}(\mathrm{~ms})$ & $P_{w 2}$ \\
\hline \multirow{2}{*}{ Case1 } & Count. transfer & $800 \mathrm{~V}$ & $100 \mu \mathrm{F}$ & 0 & $3 \mathrm{~ms}$ & - & - \\
\hline & Discount. transfer & $800 \mathrm{~V}$ & $100 \mu \mathrm{F}$ & 0 & $0.74 \mathrm{~ms}$ & $1.15 \mathrm{~ms}$ & $3 \mathrm{~ms}$ \\
\hline \multirow{2}{*}{ Case 2} & Count. transfer & $800 \mathrm{~V}$ & $100 \mu \mathrm{F}$ & 0 & $0.37 \mathrm{~ms}$ & $0.4 \mathrm{~ms}$ & - \\
\hline & Discount. transfer & $800 \mathrm{~V}$ & $100 \mu \mathrm{F}$ & 0 & $0.37 \mathrm{~ms}$ & $0.4 ; 0.6 ; 0.8 ; 1 ; 1.2 ; 1.4 ; 1.6 \mathrm{~ms}$ & $3 \mathrm{~ms}$ \\
\hline \multirow{2}{*}{ Case 3} & Count. transfer & $800 \mathrm{~V}$ & $100 \mu \mathrm{F}$ & 0 & $3 \mathrm{~ms}$ & - & - \\
\hline & Count. transfer & $253 \mathrm{~V}$ & $1000 \mu \mathrm{F}$ & 0 & $3 \mathrm{~ms}$ & - & - \\
\hline
\end{tabular}

In the beginning, one switch with a large Pw time ensuring an uninterrupted current throughout the range time was turned on. Zero points of coil current for this case allow us to determine the off-times of the first and the on-times of the second switch. First, the current waveforms for the normal operation of the bidirectional switch and the situation when the switch during experimental measurements was turned off after the fourth pulse current (counting positive and negative pulses), and then got turned on with a delay of two pulses (Figure 9) were compared (Case 1).

The model in the Maxwell program allows us to determine the volume forces acting on each element into which the disc is split. Due to the cylindrical symmetry, all the forces acting on the disc have only two components: radial and axial. On the other hand, from the viewpoint of the movement of the rigid body, the resultant force has an axial component only. Figure 10 shows the waveforms of the resultant axial forces and displacement corresponding to the continuous and discontinuous waveforms, i.e., the coil currents of Figure 9 (Case 1). Slight differences in the waveforms of these forces actually explain the lack of differences in displacement waveforms (Figure 10) and hence also the speeds. For Case 1, Figure 9 additionally presents the waveform of the capacitor voltage, which shows that at the instant of current interruption, the capacitor had already given away $99 \%$ of the initial energy. The remaining $1 \%$, which corresponds to the voltage $80 \mathrm{~V}$ of the capacitor, as it turns out, has no significant impact on the force and hence on the displacement and speed of the disc. 


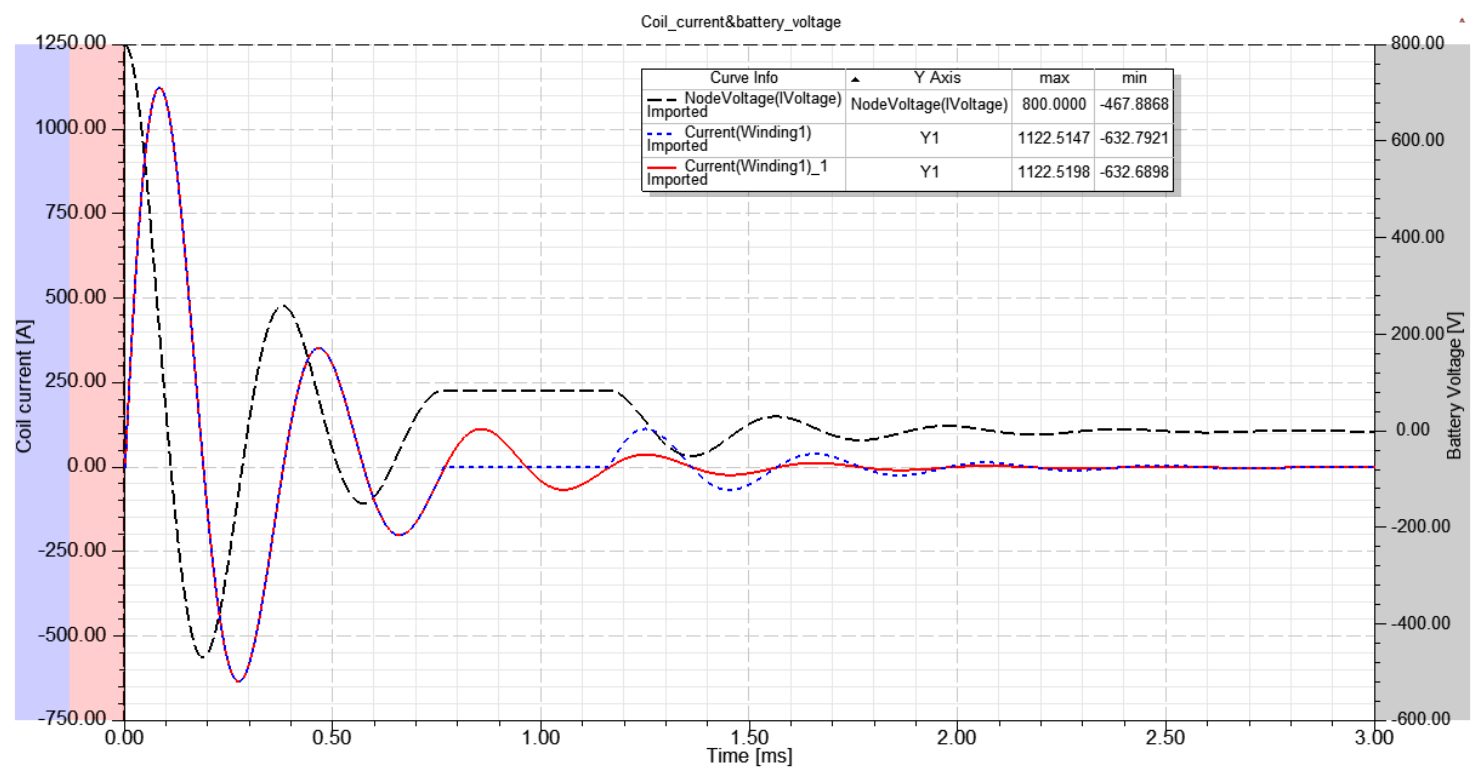

Figure 9. Continuous and discontinuous coil current and battery voltage ( $U c=800 \mathrm{~V})$, (Case 1 from Table 2).

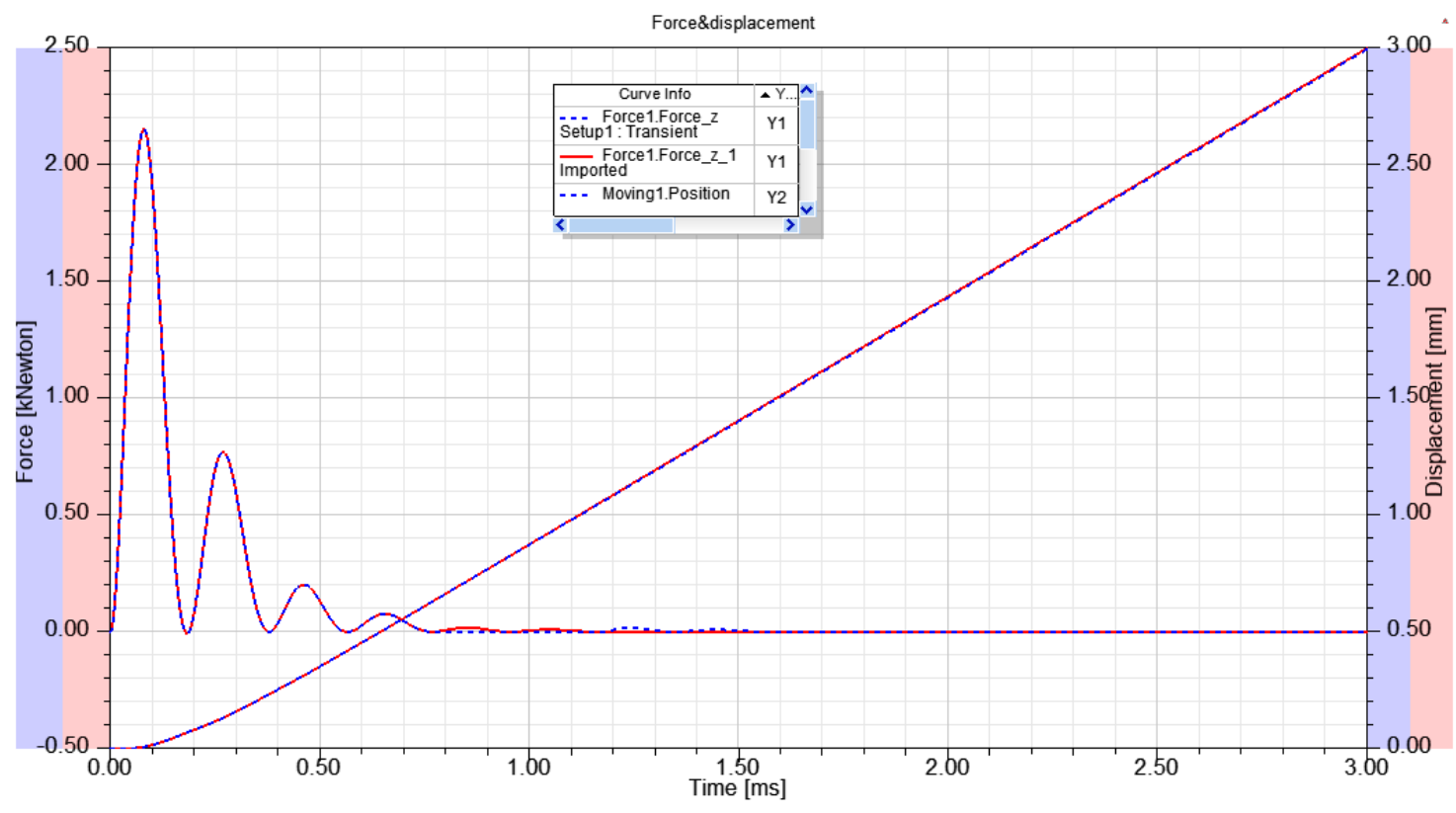

Figure 10. Resultant forces and displacements in continuous and discontinuous energy transfer (Case 1 from Table 2).

Given such a small amount of remaining energy in the capacitor, additional simulations determining the family of characteristics for the changing on-time $\left(\mathrm{Td}_{2}\right)$ of the second switch were performed (Figure 11), (Case 2 from Table 2). Figure 11 shows a comparison of coil currents and corresponding displacements. This time one can see slight differences between the displacement waveforms, where the relative difference in the maximum range that the disc achieves after $3 \mathrm{~ms}$ between continuous and discontinuous cases does not exceed $4.5 \%$. 


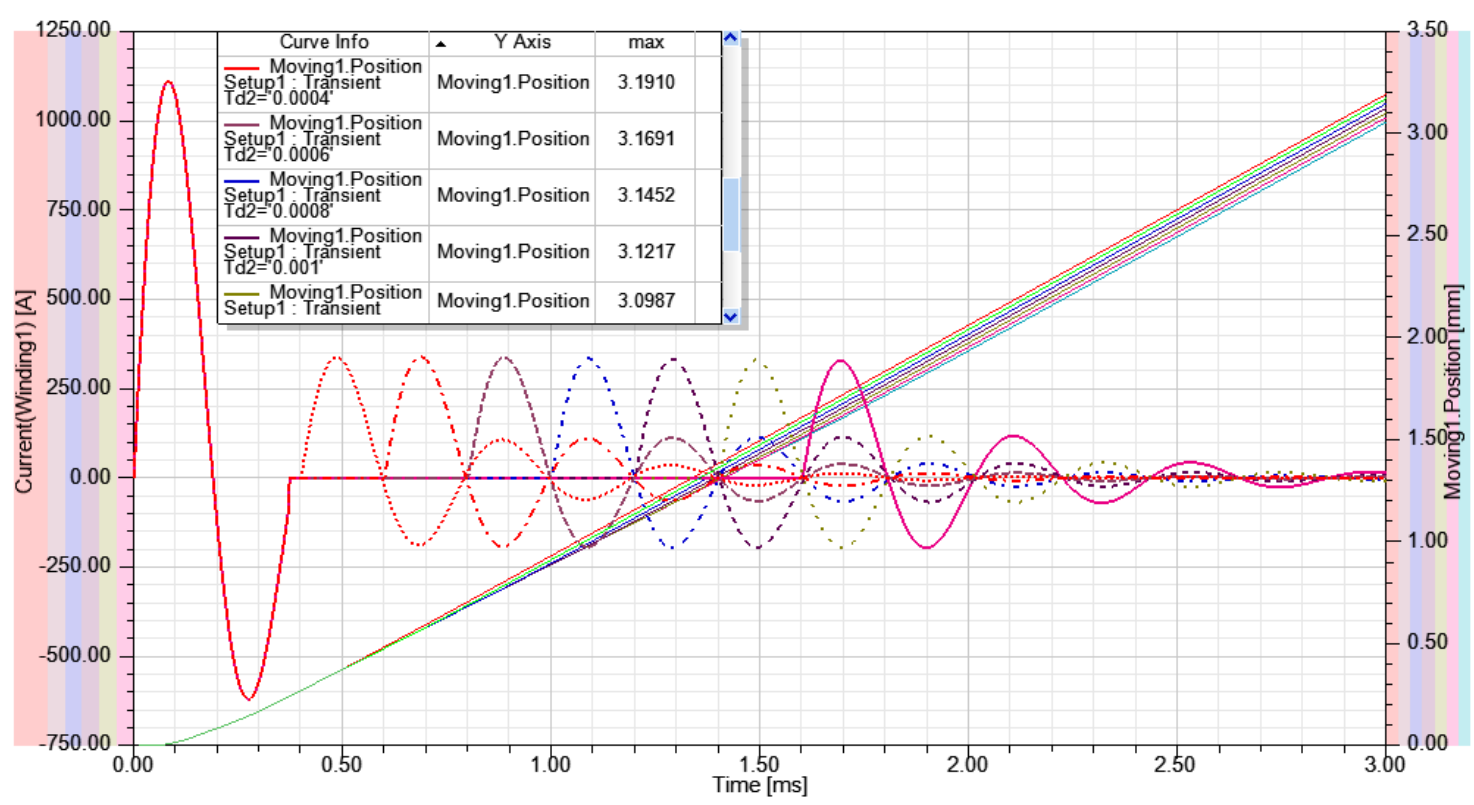

Figure 11. Coil current family and displacements corresponding to it ( $\left.U_{c}=800 \mathrm{~V}\right)$, (Case 2 from Table 2).

The circuit hybrid breakers require reaching an adequate gap between the contacts, which must ensure irreversible turning off of the circuit in which the short-circuit took place. It is most often required that the distance between the contacts obtained in suitable time amounts to approximately $3 \mathrm{~mm}[19,20]$. Generally, the aim is to make this time as short as possible. Finally, displacement trajectories were compared for two clearly differing capacitances $(C=100 \mu \mathrm{F}$ and $C=1000 \mu \mathrm{F})$. The result of the simulation from Figure 12 confirms (experimental results from Figure $5 b$ ) that in the further part of the displacement, the greater range is reached by the waveform for a greater capacitance of the battery, despite the same initial energy of the capacitors. This effect undoubtedly results from the stronger influence of the skin effect for this configuration of the capacitor battery.

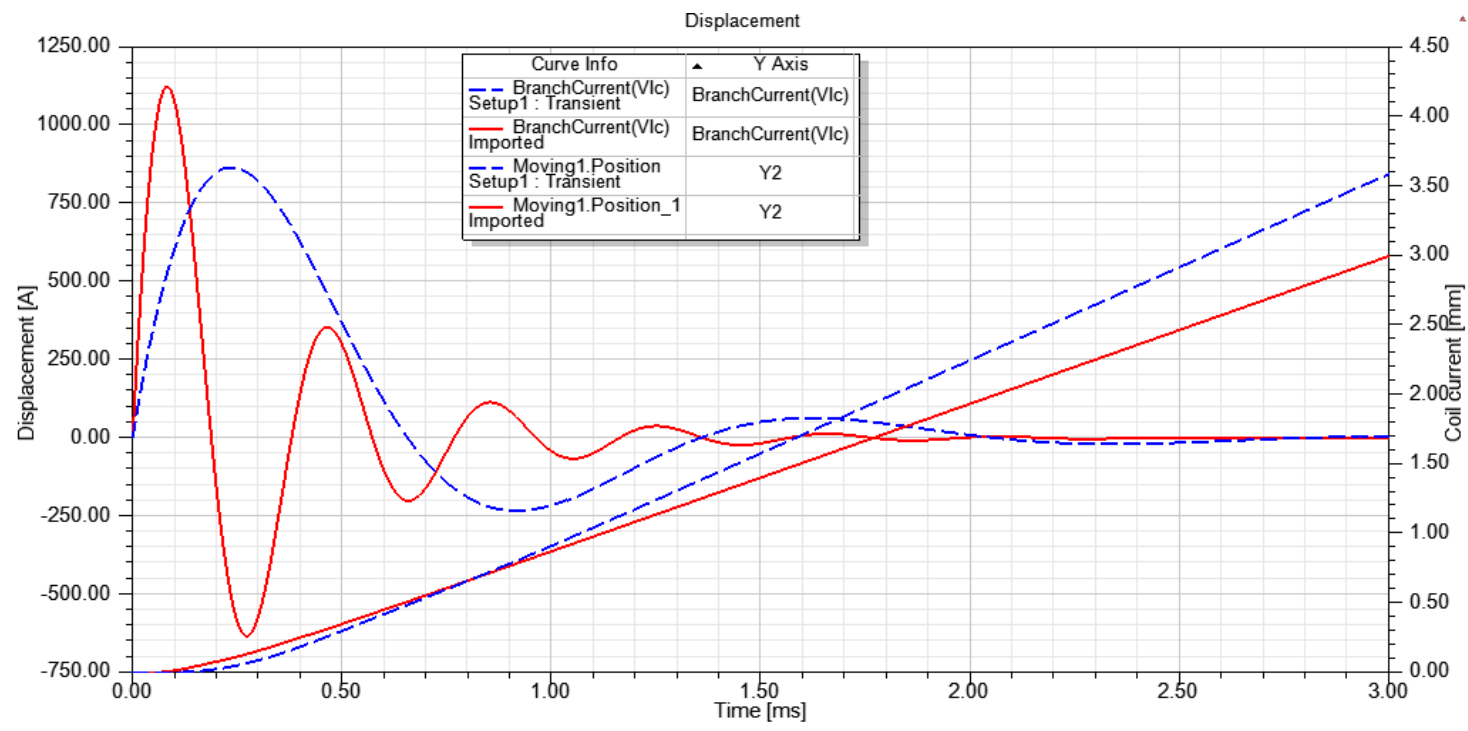

Figure 12. Coil current and disc displacements $(C=100 \mu \mathrm{F}$ red colour, $C=1000 \mu \mathrm{F}$ blue colour), (Case 3 from Table 2). 


\section{Final Remarks}

\subsection{Results and Discourse}

Commenting on the most important results of the experimental studies and simulation research, some observations are worth emphasizing. Firstly, the experimental studies-IDD—showed unambiguously that the reason for the discontinuity of energy transfer from the capacitor to the coil in the tested system are not too slow valves (thyristor or transistor), but the disruption of the gate signal, which is difficult to eliminate when significant short circuits appear, especially in the presence of environmental disturbances.

Secondly, in order to investigate the impact of discontinuities occurring at any random moment, a series of simulations was carried out in a circumferential-field model implemented in the Maxwell program. Simulation tests showed that if the delay in re-switching the valve does not exceed (for the considered $C=100 \mu \mathrm{F}$ case) $1 \mathrm{~ms}$ time, the discontinuity of energy transfer has lesser impact than the change of $2 \%$ of the achieved maximal disc reach (in a given time) (Figure 11).

Thirdly, this fact was also confirmed by experimental studies comparing the displacement of the disc in the case of continuous and discontinuous energy transfer for $100 \mu \mathrm{F}$, which was only slightly different. This means that the advantage of the displacement reach for $1000 \mu \mathrm{F}$ capacity with the same initial energy capacitor was not the result of discontinuities in energy transfer (Figures 5 and 12).

\subsection{Conclusions}

Through the experimental studies and simulation research, several conclusions can be drawn, as shown below. An important conclusion obtained as a result of both the experimental and simulation research is the demonstration of the superiority of the disc's dynamic properties (maximum displacement achieved) for higher capacitance of the capacitor battery in IDD, despite maintaining their identical initial energy. This means inferior IDD efficiency in the case of smaller capacity. An additional cause is undoubtedly a stronger skin effect that has an impact on a larger increase in resistance in the system at smaller capacities. In consequence, we would like to recommend that the designers, users and researchers connected with and interested in the considered field of investigation not only to take great care in the implementation of the anti-interference protection of the gate circuits, but also to choose capacitor with a large capacitance, because it is then more likely that the disruption will disappear before the thyristor is switched back on.

Finally in conclusion it should be emphasized that the field-circumferential propulsion model presented in this article does not take into account the deformation and vibrations of the disc and it could be a subject of further research.

\subsection{Further Studies}

When designing the propulsion it is very important to perform stress analysis of specific dimensions of the coil and disc so as not to exceed the stresses, which could lead to its permanent deformation [21]. In turn, the stress distribution in the disc is affected not only the by the maximum force value, but also by the space-time distribution of current density and force [12,18]. Therefore, the presented model will be further examined by taking into account the stress analysis for the forces generated by the continuous and discontinuous transfer of energy from the capacitor.

Acknowledgments: The studies were carried out in the framework work No WE/DS/429/2016 and financed from the funds for science by the Polish Ministry of Science and Higher Education.

Author Contributions: Piotr Jankowski and Janusz Mindykowski elaborated together a concept of the paper; Piotr Jankowski (author of models) carried out simulations and experiments; Janusz Mindykowski revised and analyzed the results.

Conflicts of Interest: The authors declare no conflict of interest. 


\section{Nomenclature}

\begin{tabular}{|c|c|}
\hline CLID & Current Limiting Interrupting Device (ultra-rapid hybrid breaker) \\
\hline IDD & Inductive Dynamic Drive (actuator) \\
\hline PE & Power Electronics Element \\
\hline CS & Contacts \\
\hline CDS & Short Circuit Current Detector \\
\hline $\mathrm{EE}$ & Element limiting the current \\
\hline$L_{z}$ & short circuit inductance \\
\hline$R_{z}$ & short circuit resistance \\
\hline$C_{g}$ & capacitor capacitance of thyristor gate (trigger) circuit \\
\hline C & total capacitance of the capacitor battery \\
\hline Uc & initial voltage value of the capacitor \\
\hline$L_{\text {coil }}, L_{c}$ & coil circuit inductance \\
\hline$R_{c}, R_{\text {coil }}$ & coil circuit resistance \\
\hline$L_{d}$ & equvivalent disc inductance \\
\hline$R_{d}$ & equvivalent disc resistance \\
\hline$M(x)$ & variable mutual inductance \\
\hline E & initial energy value of the capacitor \\
\hline$x(t)$ & disc displacement vs time \\
\hline$F(t)$ & electrodynamic axial force acting on the disc vs time \\
\hline$J(t)$ & disc current density vs time \\
\hline$B(t)$ & flux density generated by the coil current \\
\hline$F_{\text {load }}$ & motion resistance force \\
\hline $\mathrm{m}$ & aluminum disc mass \\
\hline$R i_{-c o i l}$ & inner coil radius \\
\hline Ro_coil & outer coil radius \\
\hline$h-$ coil & coil thickness \\
\hline$N$ & wires number \\
\hline Ri_disc & inner disc radius \\
\hline Ro_disc & outer disc radius \\
\hline$h \_$disc & disc thickness \\
\hline$k_{\text {skin_eff }}$ & skin effect ratio \\
\hline$r_{c}(c i)$ & radius of $c i$-th wire \\
\hline$d_{\text {wire }}$ & wire diameter \\
\hline$\omega$ & current angular frequency \\
\hline$\gamma_{c u}$ & copper conductivity \\
\hline$b_{0},\left(b_{1}\right)$ & absolute value of the modified first (second) kind Bessel function \\
\hline$\beta_{0},\left(\beta_{1}\right)$ & argument of the modified first (second) kind Bessel function \\
\hline $\mathrm{T}_{\mathrm{d}}$ & delay time of the triggering signal \\
\hline $\mathrm{T}_{\mathrm{r}}$ & rise time of the triggering signal \\
\hline $\mathrm{T}_{\mathrm{f}}$ & fall time of the triggering signal \\
\hline $\mathrm{P}_{\mathrm{W}}$ & duration of pulse of the trigger signal \\
\hline S_ON & switch \\
\hline $\mathrm{R}_{\text {off }}$ & switch resistance value \\
\hline $\mathrm{R}_{\text {on }}$ & switch resistance value \\
\hline
\end{tabular}

\section{References}

1. Nquyen, V.; Son, H.; Kim, T.N.H.; Kim, C.I. A novel topology of hybrid HVDC circuit breaker for VSC-HVDC application. Energies 2017, 10, 1675. [CrossRef]

2. Xue, S.; Lian, J.; Qi, J.; Fan, B. Pole to fault analysis and fast protection scheme for HVDC based on overhead transmission line. Energies 2017, 10, 1059. [CrossRef] 
3. Mokhberdoran, A.; Carvalho, A.; Silva, N.; Leite, H.; Carrapatoso, A. A new topology of fast solid-state HVDC circuit breaker for offshore wind integration applications. In Proceedings of the 17th European Conference on Power Electronics and Applications (EPE'15 ECCE-Europe), Geneva, Switzerland, 8-10 September 2015; pp. 1-10.

4. Tarasiuk, T.; Gorniak, M. Load Sharing in Ship Microgrids under Nonsinusoidal Conditions-Case Study. IEEE Trans. Energy Convers. 2017, 32, 810-819. [CrossRef]

5. Niwa, Y.; Yokokura, K.; Matsuzaki, J. Fundamental investigation and application of high speed VCB for DC power system of railway. In Proceedings of the 24th International Symposium on Discharges and Electrical Insulation in Vacuum, Braunschweig, Germany, 30 August-3 September 2010; pp. 125-128.

6. Alferov, D.; Budovsky, A.; Evsin, D.; Ivanov, V.; Sidorov, V.; Yagnov, V. DC vacuum circuit-breaker. In Proceedings of the 23rd International Symposium on Discharges and Electrical Insulation in Vacuum, Bucharest, Romania, 15-19 September 2008.

7. Jankowski, P.; Dudojć, B.; Mindykowski, J. Simple method of dynamic displacement record of contacts driven by inductive dynamic drive. Metrol. Meas. Syst. 2009, XVI, 5-18.

8. Jankowski, P.; Mindykowski, J. Measurement of quantities characterizing the properties of an inductive dynamic drive. Electrical Review. Przegląd Elektrotechniczny 2012, 88, 78-82.

9. Jankowski, P. Analytic-numerical approach in modelling electrodynamic phenomena of inductive dynamic drive. J. Chin. Inst. Eng. 2015, 79-86. [CrossRef]

10. Kondratyev, V.A.; Solovyev, A.L. Research of electromagnetic forces electromagnetic actuator at fluctuations of anchor. In Proceedings of the 2016 13th International Scientific-Technical Conference on Actual Problems of Electronics Instrument Engineering (APEIE), Novosibirsk, Russia, 3-6 October 2016; Volume 3. [CrossRef]

11. Ahn, H.; Lee, J.; Lee, B.; Hahn, S. Optimal design of permanent magnet actuator for vacuum circuit breakers using response surface methodology. Int. J. Appl. Electromagn. Mech. 2014, 45, 503-509. [CrossRef]

12. Jankowski, P.; Woloszyn, M. Suitability study of hybrid model of electrodynamic actuator. Int. J. Appl. Electromagn. Mech. 2014, 45, 649-657.

13. Carlstedt, M.; Porzig, K.; Uhlig, R.; Zec, M.; Ziolkowski, M.; Brauer, H. Application of Lorenz force eddy current testing and eddy current testing on moving nonmagnetic conductors. Int. J. Appl. Electromagn. Mech. 2014, 45, 519-526. [CrossRef]

14. Griffiths, D. Introduction to Electrodynamics; No. 07458; Prentice-Hall, Inc.: Upper Saddle River, NJ, USA, 2009.

15. Jankowski, P.; Mindykowski, J.; Woloszyn, M. Effect of Power frequency on the stress state of disc actuator. Int. J. Appl. Electromagn. Mech. 2014, 45, 639-647. [CrossRef]

16. Moaveni, S. Finite Element Analysis Theory and Application with Ansys, 3rd ed.; Prentice Hall: Upper Saddle River, NJ, USA, 2007.

17. Tickoo, S. Ansys for Desiners; CADCIM Technologies: Schererville, IN, USA, 2009.

18. Jankowski, P. Modelling of Magneto-Elastic Phenomena in Inductive Dynamic Drive. J. Electr. Eng. Technol. 2017, 12, 1073-1081. [CrossRef]

19. Tan, Y.; Yang, K.; Geng, Y.; Liu, Z.; Yanabu, S. Investigation on the characteristics of a high speed repulsion mechanism for wide-range operation of resistive type SFCL. In Proceedings of the 2014 International Symposium on Discharges and Electrical Insulation in Vacuum, Mumbai, India, 28 September-3 October 2014. [CrossRef]

20. Meyer, J.M. A DC hybrid circuit breaker with ultra-fast con-tact opening and integrated gate-commutated thyristors (IGCTs). IEEE Trans. Power Deliv. 2006, 21, 646-651. [CrossRef]

21. Pasca, S.; Vesselenyi, T.; Mudura, P.; Tomse, M.; Monica, P.; Firetenau, V.; Tudorache, T. Electromagnetic Forming an Efficient Technology for Metallic Sheet Processing. Electrical Review. Przeglad Elektrotechniczny 2008, 84, 197-202.

(C) 2018 by the authors. Licensee MDPI, Basel, Switzerland. This article is an open access article distributed under the terms and conditions of the Creative Commons Attribution (CC BY) license (http:/ / creativecommons.org/licenses/by/4.0/). 